\title{
Solar-Based Thermoelectric Generator and its ANFIS Model
}

\author{
Ali Ekber Özdemir \\ Fatsa Faculty of Marine Sciences, Ordu University, 52400, Ordu, Turkey \\ a.ekber@odu.edu.tr
}

Received: 7 May 2018

Accepted: 18 June 2018

DOI: $10.18466 /$ cbayarfbe. 421575

\begin{abstract}
In this work, it is aimed to construct an Adaptive Neuro Fuzzy Inference System (ANFIS) model using the experimental values of our previous work on solar heating with wind chimney thermoelectric generator and to predict the generated open circuit voltage of experimental system under variable conditions. The ANFIS model constructed makes use of input parameters such as local radiation intensity on solar collector tube (W), ambiance temperature ${ }^{\circ} \mathrm{C}$ and average wind velocity in the chimney $(\mathrm{m} / \mathrm{s})$. Open circuit voltage $(\mathrm{V})$ is denoted as output. Selected experimental data sets are used in training and testing procedures to accomplish the model required. Assessment of the outcomes of the study reveals that the proposed modeling by ANFIS is consistent and validated by the experimental results. Promising results show that ANFIS model can be used to estimate the output parameter of solar-based generator (the open circuit voltage) correctly and this result can use enhancing of presented system. Employment of artificial neural networks on renewable energy systems is a rather new area of study. Hence, continuing work via neural network structures will be related to the optimization and improvement of these generators for useful energy producing.
\end{abstract}

Keywords: Energy conversion, Fuzzy neural networks, Renewable energy sources, Solar heating, Thermoelectricity.

\section{Introduction}

Energy is an indispensable element of economic and social development. It is important to ensure the continuity of energy for sustainable development. Due to this reason, developed countries are in search for new energy systems. Nowadays, energy requirement is ever increasing so energy needs of the countries are growing rapidly for economic and social industries.

Though the energy demand is continuously increasing in the world and proportionate decrease of fossil fuels, and consequently increase in environmental problems has been faced. Hence, clean energy sources such as solar, wind, hydropower, geothermal and marine energies have become indispensable for environmental future of the world due to their emission nature [1].

Nowadays, many investments are under way to accomplish the energy demand. Worries about environmental problems direct researchers to the renewable and clean energy areas. Also development of new technologies in this area is important and this affects the applications positively. Our previous work can be given as an example to such applications [2].

It is known that artificial neural network (ANN) methods are used in renewable energy applications frequently. In the literature, usage of ANN methods in renewable energy applications usually divides three basic branches. These can be given as following.
- Making estimation or modeling the potential of renewable energy sources [1 - 6].

- Making estimation of power production in renewable energy system [7 - 10].

- Modeling, analyzing or optimizing of energy producing system [11 - 14].

The aim of the presented work is to create an ANFIS model of constructed experimental system. It is advantageous to model the virtual energy system. Therefore, for optimization of the constructed systems it is required to know the effects of the variables on the systems. This can be achieved using reliable models without troublesome experiments. It is possible to test limits of constructed system against under different conditions and to make the cost analysis of system under the variable conditions for a selected time period by using a virtual model. Also, making a choice of geographical location without any test is a potential way.

In the previous work published by the authors [2], parametric factors such as solar radiation, wind velocity, ambient temperature that affect the system area dependent on geographical location of the constructed system. Finally, it is possible, by employing geographical location and climatological data, to assess an investment as to its goodness by using a virtual model. Based on this given knowledge, the ANFIS model presented here is used to predict the generated open circuit voltage under variable parametric values. In this presented work, the 
experimental system which is constructed with a wind chimney, TE module, heat sink, evacuated tube and thermosiphon type heat pipe [2] is analyzed by an ANFIS model.

Thermoelectric generator (TEG) modules have been of great interest to achieve renewable energy. These modules have no mechanical moving parts, and are sufficiently ergonomic, light, quiet and eco-friendly DC sources [2 - 3]. The working principle of the Thermoelectric (TE) module is based on Seebeck effect that was discovered in 1821 by Thomas Johan Seebeck. They have been used in many areas where the thermal flux exists in both commercial and private sectors.

The efficiency of the TE module is related to the thermal efficiency of the device that depends on temperature difference between the sides of the TE module [4]. Upon a consideration of all these advantages, TEGs have been used in aerospace and military applications in past years [5]. It is also considered for the waste heat recovery TEG, such as power plants, geothermal energy, heat engine and other industrial waste heat generation processes [2], [5 7].

TE energy generation is a direct conversion of thermal energy to electrical energy [8]. When the temperature gradient exists between the sides of the module, an electrical voltage is generated to supply an external electrical load.

Applications of these modules are not sufficiently widespread because of the low efficiency (typically less than 5\%) [6] and high cost [8]. TEGs available in the market are produced for preferred purposes and areas and with optimized performance.

The TEG module contains semi-conductor materials that are thermally in parallel but electrically in series. Generally, hot surface of the TE module can withstand application range about 300 for use in practical thermal applications [9] and the application range between $77{ }^{\circ} \mathrm{C}$ $(350 \mathrm{~K})$ and $177{ }^{\circ} \mathrm{C}(450 \mathrm{~K})$ has attracted interest considerably [10]. Performance of TEGs which can withstand $800{ }^{\circ} \mathrm{C}[11$ - 12] have also been reported.

In the next section, the modeled system is described. Afterwards the related ANFIS model will be described in detail. After a description of general structure of ANFIS, the ANFIS model constructed with input and output parameters in this work will be presented. Results of the ANFIS model dependence on input and output variables will be presented. The results will be discussed in conclusion section.

\section{Materials and Methods}

In our paper, modelled system is based on thermoelectric energy conversion. And main part of the system is a TEG module. A TEG module converts thermal energy into electrical energy. The working principles of a TEG module is based on Seebeck effect. TEGs are consisting of $\mathrm{p}$ and $\mathrm{n}$ type semiconductor material that are electrically in series and thermally in parallel. Structure of a TEG module can be seen in Figure 1.

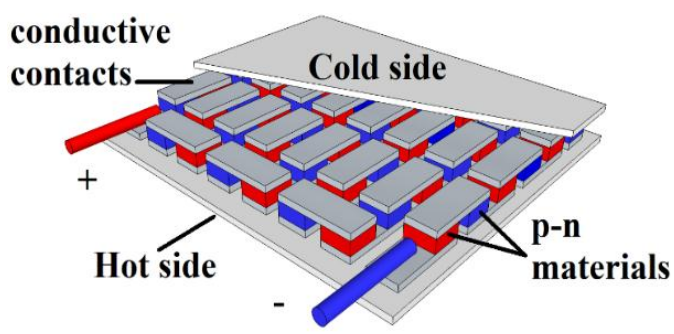

Figure 1. Structure of a standard TEG module.

Some electrical quantities such as Seebeck coefficient $(\alpha T E G)$, maximum output power (Pmax) and electrical

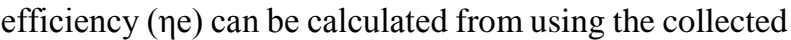
data from experimental constructions.

General structure of modelled system is shown in Figure 2. Figure 2 (a) shows physical structure and Figure 2 (b) shows schematic diagram of our experimental construction. In addition, presented system is modeled [2].
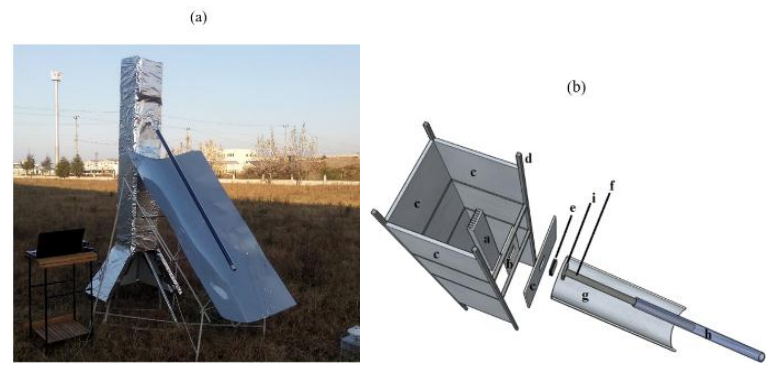

Figure 2. (a) Physical structure and (b) schematic diagram of experimental setup.

a) Aluminum block for cooling process

b) Isolation material for thermal isolation to aluminum block from metal body of wind chimney

c) Isolation material for thermal isolation to metal body of wind chimney from solar radiation

d) Metal body of wind chimney

e) TE module

f) Heat pipe

g) Reflector

h) Solar collector tube

i) Head of heat pipe

Solar collector tube consists of evacuated glass tubes which is $1.8 \mathrm{~m}$ length, $37 \mathrm{~mm}$ inner diameter with 0.209 $\mathrm{m} 2$ surface area. A parabolic mirror that is located under the tube with dimensions $1 \mathrm{~m} \times 2 \mathrm{~m}$, reflects the sun light towards the solar collector tube. Used chimney is play an important role for cooling process. Chimney has a cross- 
sectional area of $0.27 \times 0.27 \mathrm{~m}$ and length of chimney is $2 \mathrm{~m}$.

One of the most important points is thermal flux on the thermoelectric generator for constructed system. Thermal flux plays an important role on efficiency of proposed system. Calculated maximum electrical efficiency of proposed system is $0.459589 \%$ [2]. To create enough thermal flux for generating electricity, many components were used as shown in experimental setup $(\mathrm{a}-\mathrm{i})$.

\subsection{Methods}

In general, artificial neural networks are based on the mathematical model of human brain. Although there are many kinds of neural network types, all of them are based on the imitation of real neural cells. Nowadays, these artificial neural network types are used for applications on system modeling, parameter estimation, curve fitting, classification, optimization etc. [13-17]. ANFIS is well known as a special kind of artificial neural network and is based on fuzzy inference system. It was developed in 1993 by Jang [18]. This neural network structure consists of 5 layers. These layers and the general structure of ANFIS are shown in Figure 3.

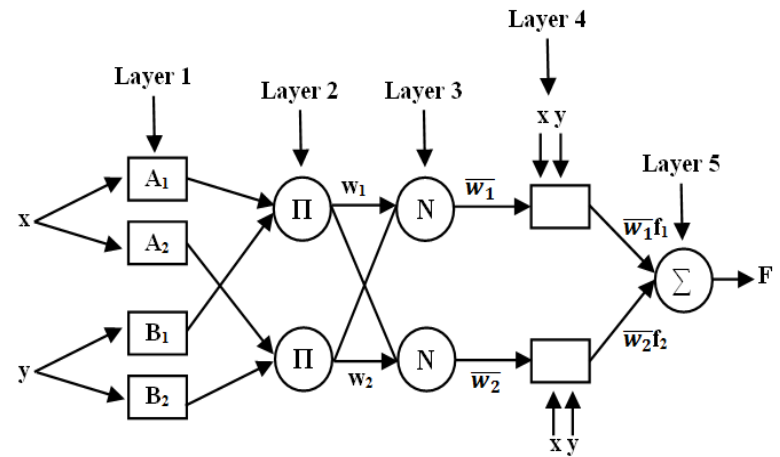

Figure 3. ANFIS structure and its layers.

An ANFIS structure that uses SUGENO type fuzzy inference system can be explained on the system that consists of two input data (x,y) and one output data $(\mathrm{F})$, as follows:

These layers are explained with equations mentioned in [18]:

Layer - 1: Membership values are obtained from the comparison of inputs with the membership functions of each rule. This process is carried out in first layer of ANFIS and this layer is called the fuzzifier layer and is shown in Figure 4.

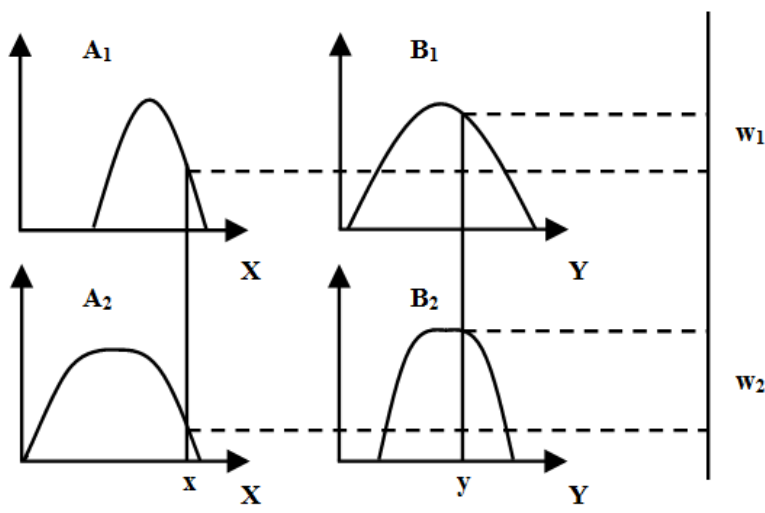

Figure 4. Fuzzifier layer.

Layer - 2: These membership values are combined with some operators (multiplication, max, min etc.). This process is carried out in the second layer of ANFIS. This layer is called the rule layer.

$$
w i=A i(x) \cdot B i(y)
$$

Layer - 3: Outputs of the second layer are normalized in the third layer. Therefore, this layer is called the normalization layer.

$$
\overline{W_{l}}=\frac{W_{i}}{\sum W}
$$

Layer - 4: The normalized values from the third layer are weighed as inputs in the fourth layer.

$$
\bar{W}_{l} f_{i}=\bar{W}_{l}\left(p_{i} x+q_{i} y+r_{i}\right)
$$

Layer - 5: In the last layer all outputs of fourth layer are summed and finally the ANFIS output is obtained.

$$
F=\sum \bar{W}_{\imath} f_{i}=\sum \bar{W}_{l}\left(p_{i} x+q_{i} y+r_{i}\right)
$$

After the last layer a polynomial output is obtained and the training stage aims to find independent parameters of this polynomial output. There are many training algorithms used for this purpose [19 - 20]. Generally, some different algorithms are used together for training of ANFIS. In our work we used hybrid algorithm [18] for the training of ANFIS.

In this work, all obtained parameters such as, local radiation intensity on solar collector tube (W), ambiance temperature $\left({ }^{\circ} \mathrm{C}\right)$ and average wind velocity in the chimney $(\mathrm{m} / \mathrm{s})$ are used on ANFIS.

The experimental data was collected on 15th, 16th, 19th, 20th and 21st of August. For constructing the ANFIS model, the experimental data of the first four days were used for the training process (15th, 16th, 19th and 20th of August) and experimental data of the last day (21th of August) was used for the testing process. 
The collected data that belongs to hot and cold side temperatures of the TE module, solar intensity on pyranometer, ambiance temperature and wind velocity in the chimney, open circuit voltage and short circuit current measurements were recorded in fifteen minutes' intervals and collected between 8:30 a.m. and at 4:00 p.m within the experimental days.

The parametric values given below (Figs.5 - 9) prepared with chronological order as data index. The mentioned data index in the figures belongs to time period of fifteen minutes' intervals. So, all used parametric values that are modelled with ANFIS are normalized values (between 0 and 1).

In modeling, input parameters are local radiation intensity on solar collector tube (W), ambiance temperature $\left({ }^{\circ} \mathrm{C}\right)$ and average wind velocity in the chimney $(\mathrm{m} / \mathrm{s})$. In this case, open circuit voltage $(\mathrm{V})$ is used as output.

Collected data set are shown in Figure.5, Figure.6, Figure.7, Figure.8 and Figure.9, respectively.

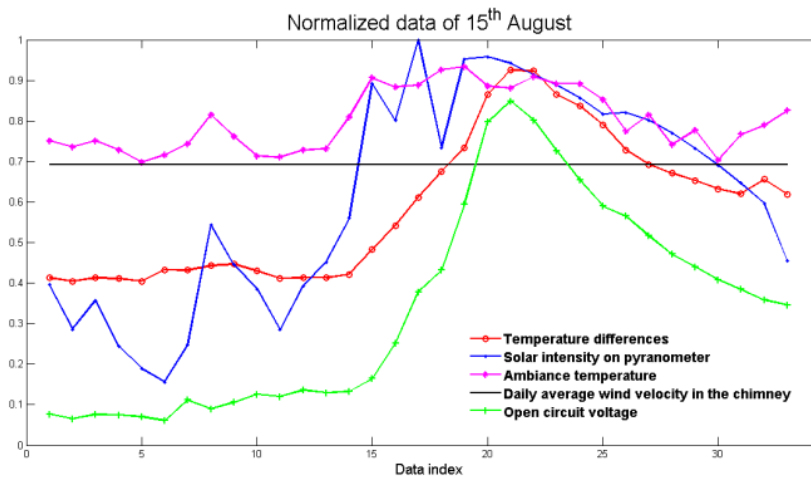

Figure 5. Collected data on 15th August.

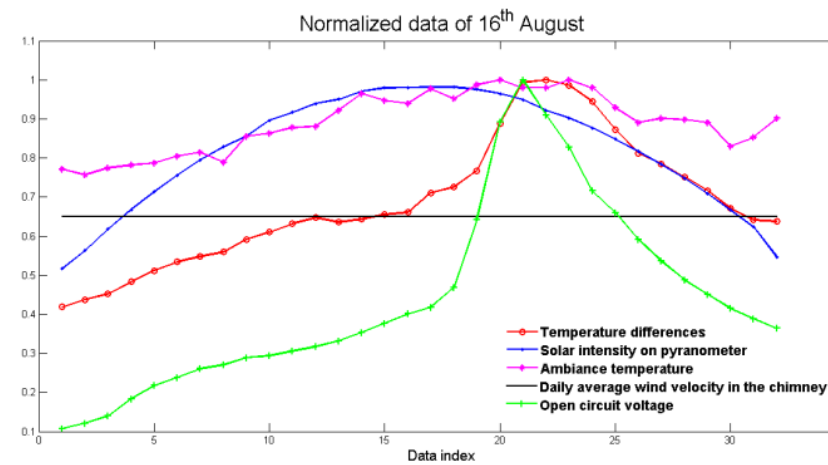

Figure 6. Collected data on 16th August.

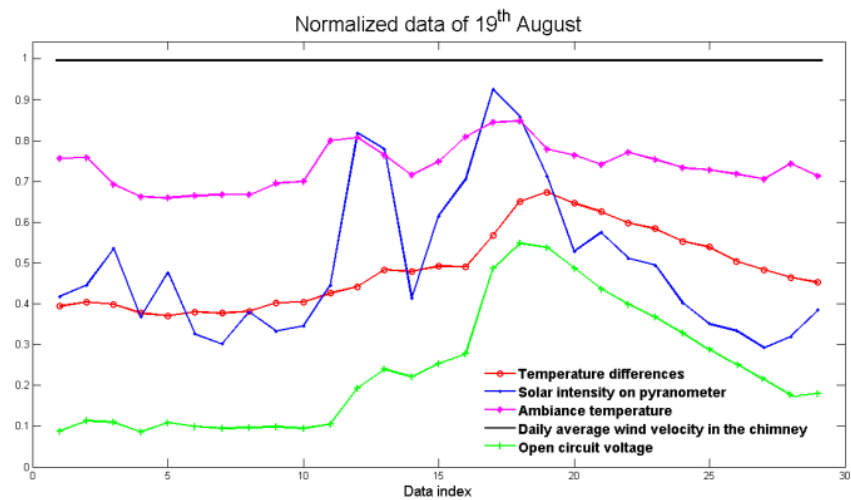

Figure 7. Collected data on 19th August.

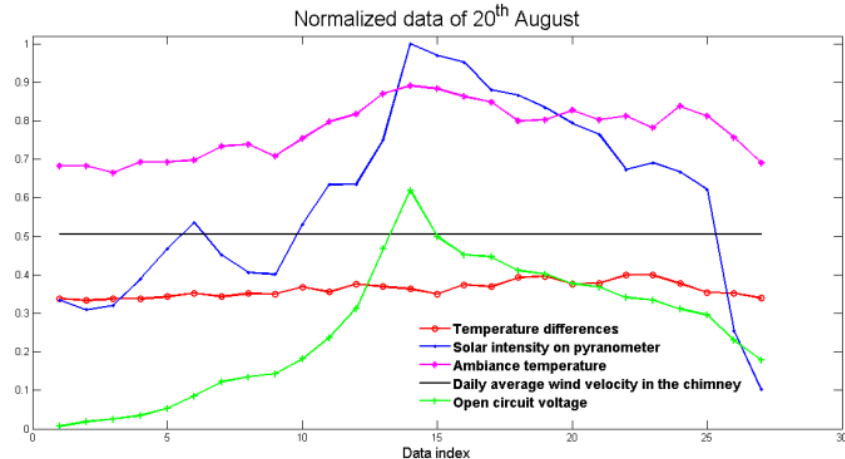

Figure 8. Collected data on 20th August.

develop

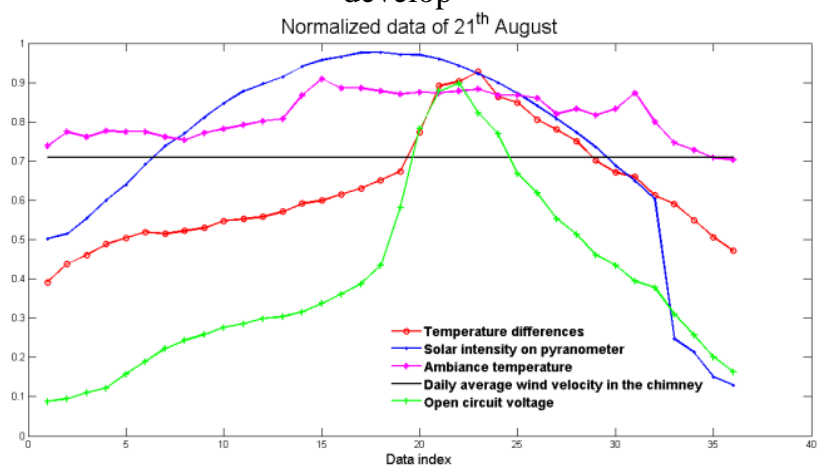

Figure 9. Collected data on 21th August.

Upon analyses of these figures, it is seen that many different parameters have been used for ANFIS modeling. For instance, in Figure. 4 the variation of solar density shows that the weather was partially cloudy on the 15th of August. As a result of this, the sun was shading and the solar intensity was decreasing distinctly by the time. For all that, in Figure. 5 that belongs to the 16th of August, given parameters show that weather condition was clearly sunny.

In Figure.7, it is seen that the values of the average wind velocity on that day are greater than those on the other days. When collected parametric values were analyzed particularly, it is noticed that the most variable parameter was the wind velocity for all days. This variation is used as ill condition for modeling. In this way the average of the wind velocity values for an experimental day were used. Accordingly, it is seen from Figure.8, related to 20 
August, all temperature values are comparably low.

The constructed ANFIS model of the presented generator is shown in Figure.10 with the input and output parameters.

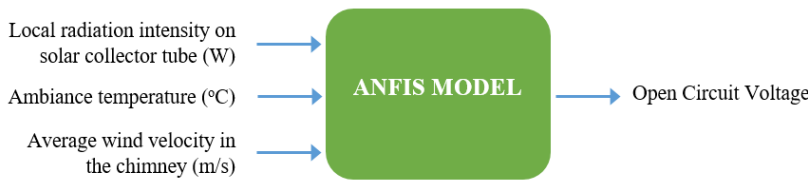

Figure 10. Inputs and output of the constructed ANFIS model.

ANFIS is a rule based neural network. In this work the ANFIS rules have been selected with grid partition and the training algorithm of ANFIS have been selected as hybrid training.

The membership function (MF) has been selected as the generalized bell (gbell) function and for each input grid size as trials 2, 2, 1 respectively. With selected grids, $2 \times 2 \times 1=4$ rules were constructed. ANFIS structure of presented work is shown in Figure.11.

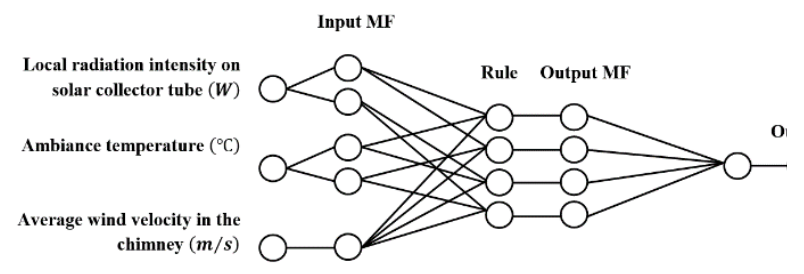

Figure 11. The constructed ANFIS structure.

\section{Results and Discussion}

Measured experimental data are normalized and used for training and testing processes. Total size of data is in 155 x 4 matrix form. The measured data of the 15th, 16th, 19th, 20th of August are used for the training process and the measured data of the 21st of August is used for the testing process. Average values of training and testing error are given as root mean square error (RMSE). At the end of the training process an average training error of 0.042 and a testing error of 0.046 have been acquired. The experimental data and the output of the ANFIS for the training and testing processes are shown in Figure 12 and Figure 13.

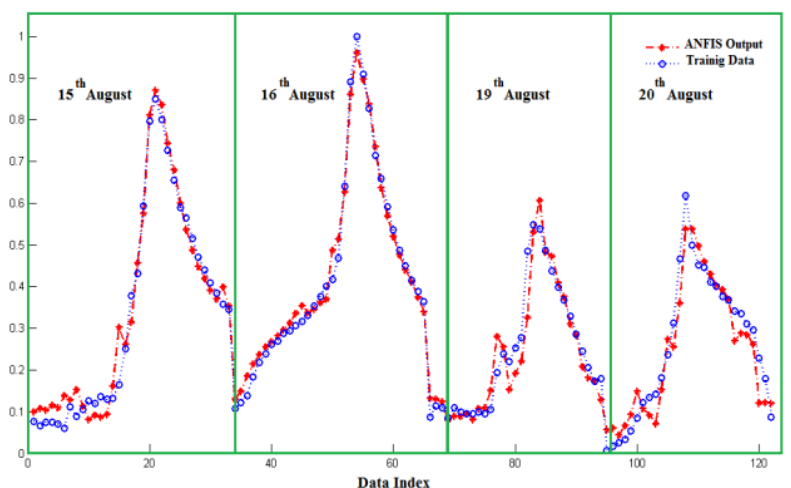

Figure 12. The experimental data and output of the ANFIS for the training process

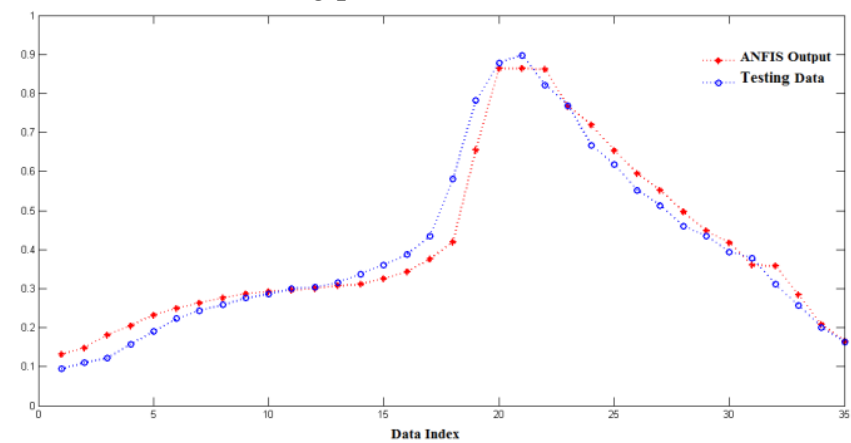

Figure 13. The experimental data and output of the ANFIS for the testing process.

For constructing an ANFIS model of the presented generator the usage of a larger number of rules is attempted. It is seen that a larger number of rules decreases the training error but at the same time increases the testing error. For example, when 16 rules are used, the average training error is 0.0135 and the average testing error is 0.075 . But when 81 rules are used, the average training error is 0.00263 and the average testing error is 0.228 .

\section{Conclusion}

Renewable energy systems are still in the stage of development and design processes evolve accordingly. It is very important to get reliable systems for two reasons. The first one is that it is possible to estimate output data without performing troublesome experiments under several conditions. This makes the process of deciding easier on the best conditions for generating electricity without any experiment thus giving an opportunity for making an investment in a healthy way. The other one is that it is possible to perform the optimization of the constructed system.

In this work, the ANFIS model of a previous experimental system related to solar based thermoelectric generator with wind cooling chimney is studied. The constructed ANFIS model is tested to estimate the amount of generated open circuit voltage under different conditions. Usage of ANFIS is thought as a means to get the values of accessible open circuit voltage of the constructed system. When the measured experimental values of the system are used for the training and testing processes, it is observed that the obtained numerical values of artificial intelligence are in good agreement with the experimental values

The ANFIS modeling reveals that it is possible to estimate the values of the open circuit voltages at any of input parameter set of the constructed system. Our results show that the constructed ANFIS model is reliable. Therefore, it can be a usable tool for analyses such as cost analysis, investment analysis etc. by using geographical location and climatological data. 


\section{Acknowledgement}

This paper and its related research are supported by grants from the Ordu University, Department of Scientific Research Project Development Program of Turkey. (AR-1418, AR-1631 and AR-1641)

\section{References}

1. Canbing L, Haiqing S, Yijia C, Jianhui W, Yonghong K, Yi T, Jing $\mathrm{W}$, Comprehensive review of renewable energy curtailment and avoidance: A specific example in China, Renewable and Sustainable Energy Reviews, 2015, 41, 1067-1079.

2. Ali, E.Ö, Yavuz K, Engin Ö, Tahsin A, The experimental design of solar heating thermoelectric generator with wind cooling chimney, Energy Conversion and Management, 2015, 98, 127-133.

3. Zuryati D, Nandy P, Ral A.K, The utilization of heat pipe on cold surface of thermoelectric with low-temperature waste heat, Applied Mechanics and Materials, 2013, 302, 410-415.

4. Michele, L.O, Emily W, Philip A, Parilla, Eric, S.T, Cheryl, E.K Gerald, J.S, Samad, A. F, Bill, J.N, Andriy Z, Alan, A.G, Craig, S.T, Judy N, Matthew, H.G, Paul, F.N, Robert T, Lauryn, L.B, Allison G, David, S.G, A high-temperature, high-efficiency solar thermoelectric generator prototype, Energy Procedia, 2014, 49, $1460-1469$.

5. Xing N, Jianlin Y, Shuzhong W, Experimental study on lowtemperature waste heat thermoelectric generator, Journal of Power Sources, 2009, 188(2), 621-626.

6. David M.R, Thermoelectrics: an environmentally-friendly source of electrical power, Renewable Energy, 1999, 16 (1), 1251-1256.

7. Ono, K, Suzuki, R.O, Thermoelectric power generation: converting low-grade heat into electricity, Journal of the Minerals, Metals \& Materials Society, 1998, 50(5), 12-31.

8. Samir B, Mauro B, Alessandro Z, Stefania S, High efficiency Thermo-Electric power generator, International Journal of Hydrogen Energy, 2012, 37(2), 1385-1398.

9. TECTEG MFR, http://thermoelectricgenerator. com/wpcontent/uploads/2014/04/SpecTEG1-12611-6.0Thermoelectricgenerator1.pdf (Accessed 05.05.2018).

10. Narong V, Jongjit H, Joseph K, Michel D, Design and analysis of solar thermoelectric power generation system, International Journal of Sustainable Energy, 2005, 23(3), 115-127.

11. Perumal R, Narasimhan, K.R, Ruchi G, Development, design and performance analysis of a forced draft clean combustion cookstove powered by a thermo electric generator with multi-utility options, Energy, 2014, 69, 813-825.

12. Kyeongsoon P, H. K. Hwang, Jang, W.S, Wonseon S, Enhanced high-temperature thermoelectric properties of Ce- and Dy-doped $\mathrm{ZnO}$ for power generation, Energy, 2013, 54, 139-145.

13. Gökhan K, Ali, E.Ö, İlyas E, Reviewing and designing preprocessing units for RBF networks: initial structure identification and coarse-tuning of free parameters, Neural Computing and Applications, 2013, 22, 1655-1666.
14. Xiaobing K, Xiangjie L, Kwang, Y. L, Data-driven modelling of a doubly fed induction generator wind turbine system based on neural networks, IET Renewable Power Generation, 2014, 8(8), $849-857$.

15. Liu Y, Holmes P, Cohen J, A neural network model of the Eriksen task: reduction, analysis, and data fitting, Neural computation, $2008,20(2), 345-373$

16. Xue-Bin L, Jun W, A recurrent neural network for nonlinear optimization with a continuously differentiable objective function and bound constraints, IEEE Transactions on Neural Networks, 2000, 11(6), $1251-1262$

17. Ravinder, K.K, S.L. Shimi, Shantanu C, Fahim A, Modeling of solar PV module and maximum power point tracking using ANFIS, Renewable and Sustainable Energy Reviews, 2014, 33, 602-612.

18. Jang, J.S.R, ANFIS Adaptive-Network-Based Fuzzy Inference Systems, Man and Cybernetics, 1993, 23, 665-685.

19. Jang,J.S.R, Mizutani, E, Levenberg-Marquardt method for ANFIS learning, Fuzzy Information Processing Society Biennial Conference of the North American, Berkeley, CA, 1996, pp 87 91.

20. Shoorehdeli, M.A, Teshnehlab, M, Sedigh, A.K, Novel hybrid learning algorithms for tuning ANFIS parameters using adaptive weighted PSO, Fuzzy Systems Conference, London, UK, 2007, pp. $1-6$ 